\title{
Effect of Mixing During Fermentation in Yogurt Manufacturing
}

\author{
E. J. Aguirre-Ezkauriatza, M. G. Galarza-González, A. I. Uribe-Bujanda, M. Ríos-Licea, F. López-Pacheco, \\ C. M. Hernández-Brenes, and M. M. Alvarez ${ }^{1}$ \\ Centro de Biotecnología-FEMSA, Tecnológico de Monterrey, Ave. Eugenio Garza Sada 2501 sur. Monterrey, Nuevo León, México, CP 64849
}

\begin{abstract}
In traditional yogurt manufacturing, the yogurt is not agitated during fermentation. However, stirring could be beneficial, particularly for improving heat and mass transport across the fermentation tank. In this contribution, we studied the effect of low-speed agitation during fermentation on process time, acidity profile, and microbial dynamics during yogurt fermentation in 2 laboratory-scale fermenters $(3$ and 5 L) with different heat-transfer characteristics. Lactobacillus bulgaricus and Streptococcus thermophilus were used as fermenting bacteria. Curves of $\mathrm{pH}$, lactic acid concentration, lactose concentration, and bacterial population profiles during fermentation are presented for static and low-agitation conditions during fermentation. At low-inoculum conditions, agitation reduced the processing time by shortening the lag phase. However, mixing did not modify the duration or the shape of the $\mathrm{pH}$ profiles during the exponential phase. In fermentors with poor heat-transfer characteristics, important differences in microbial dynamics were observed between the agitated and nonagitated fermentation experiments; that is, agitation significantly increased the observable specific growth rate and the final microbial count of $L$. bulgaricus.
\end{abstract}

Key words: yogurt, agitation, microbial ecology, mixing

\section{INTRODUCTION}

Yogurt has a long record of beneficial attributes for human health (Elli et al., 2006; Ekinci and Gurel, 2008). Although there is still an ongoing debate on how much bacteria originally present in yogurt survives the very acidic gastric tract, yogurt is generally considered as a probiotic product (see, for example, Elli et al., 2006).

Received March 2, 2008.

Accepted July 18, 2008.

${ }^{1}$ Corresponding author: Mario.Alvarez@itesm.mx
Yogurt is commercially produced through fermentation by lactic acid bacteria (commonly Lactobacillus spp. and Streptococcus spp.) at temperatures usually in the range of 27 to $40^{\circ} \mathrm{C}$. In typical processes of elaborating yogurt, the yogurt is not agitated during the fermentation stage. The most traditional process for yogurt elaboration involves milk fermentation by lactic acid bacteria in the same containers in which the product will be distributed. The use of nonagitated small vessels is also possible. For liquid formulations or formulations mixed with fruit, mixing is applied after fermentation.

Stirring might result in benefits to the processing of yogurt, particularly by improving the mass- and heattransport conditions. During fermentation, a critical variable that influences kinetic progress (and therefore acidification and gelation rates) is temperature (see, for example, Kristo et al., 2003). Fermentation tanks for yogurt elaboration are normally small to medium sized, commonly between 1 and $3 \mathrm{~m}^{3}$. Greater volumes would be prohibitive because of the temperature gradients occurring under nonagitated conditions, which would favor a large dispersion of microenvironmental conditions for the bacteria involved in the fermentation process, consequently allowing a wide spectrum of kinetic behaviors. Oxygen concentration gradients (from the free surface to the bottom of the tank) are also conceivable under nonagitated conditions, which would induce differences in the metabolic rate of the bacteria participating in the fermentation at different tank levels. By improving heat-transfer and mass-transfer conditions, stirring could reduce temperature heterogeneity and destroy concentration gradients during fermentation, providing a more homogeneous microenvironment for bacterial growth. This might have positive economic and product quality-related benefits, such as making possible the use of larger fermentor volumes, reducing the fermentation time, and better standardizing process performance and product attributes.

In general, 3 main arguments have been stated to justify why agitation should not be used during fermentation of industrial yogurt manufacture: (a) agitation might interfere with the gel-formation process, a key quality attribute in firm yogurt (Kristo et al., 2003; 
Lee and Lucey, 2004); (b) agitation during fermentation would inhibit the lactic acid formation involved in the process (Driessen, 1998; Early, 2000), consequently extending the fermentation time and possibly altering the quality; (c) agitation could favor the incorporation of air into the system, interfering with the fermentation dynamics, an anaerobic event. The first of these concerns is related to tradition. Nowadays, there is a growing market share for liquid yogurt-like formulations, for which gel formation is not a relevant quality characteristic for customers. The remaining 2 concerns are technical observations, which, to our knowledge, have not been thoroughly evaluated.

Information on the subject of agitation or mixing of yogurt is scarce. Most contributions published have focused on the rheological aspects of yogurt systems under agitated conditions (e.g., Patocka et al., 2004; Maingonnat et al., 2005; Kulkarni et al., 2006). Here, we studied the effect of mixing during the fermentation stage of yogurt manufacturing (benign agitation at 200 rpm). Results were compared with those obtained from the typical nonagitated process. We discuss fermentation data obtained with 2 different small-scale bioreactor geometries with significant differences in their heattransfer systems. We compared different experimental scenarios in terms of the time required to complete the fermentation (final $\mathrm{pH}$ value at 4.5), $\mathrm{pH}$ and acidity profiles in time, and microbial population dynamics of Streptococcus thermophilus and Lactobacillus bulgaricus, bacteria typically used in yogurt elaboration.

Some typical characteristics of the microbiology of traditional yogurt making are well known: (a) the synergistic growth of S. thermophilus and L. bulgaricus, (b) an optimum temperature slightly above $40^{\circ} \mathrm{C}$, (c) the earlier temporal dominance of $S$. thermophilus, and (d) the later appearance of $L$. bulgaricus, when culture conditions become progressively acidic (Riva et al., 1997). However, even for traditional nonagitated manufacture, to our knowledge, reports on microbial population dynamics are scarce (see Mahdian and Mazaheri-Tehrani, 2007). In addition, although some contributions have examined the microbiology of yogurt (see, for example, Riva et al., 1997; Parayre et al., 2007; Ekinci and Gurel, 2008), no previous study has addressed the effect of agitation on the population dynamics. Potentially, a more homogeneous environment, resulting from agitation, might render conditions more favorable for the synergy between L. bulgaricus and S. thermophilus.

Indeed, only a few examples have documented the mixing effects on the population dynamics of mixed cultures in bioreactors (see, for example, Manjarrez et al., 2000; Albasi et al., 2001; Al-Homoud et al., 2007; Padmasiri et al., 2007).

\section{MATERIALS AND METHODS}

\section{Experimental Design}

Fermentation experiments were conducted at $40^{\circ} \mathrm{C}$ to investigate the effect of agitation during the fermentation stage of yogurt manufacturing in small-scale bioreactors (referred to below). Low-agitation (200 rpm) and no-agitation conditions $(0 \mathrm{rpm})$ were tested. The order of execution of the experiments was randomized. Overall process time, length of the lag and exponential phases (based on $\mathrm{pH}$ behavior), $\mathrm{pH}$ and lactic acid concentration profile in time, L. bulgaricus and S. thermophilus specific growth rate and microbial count through fermentation, viscosity, and flavor were evaluated. In the nonagitated fermentation experiments, the $\mathrm{pH}$ and temperature gradients within the bioreactor were documented by taking samples at 3 different locations and measuring temperature (model 52II Thermopar system, Fluka, Everett, WA) and pH (model $\Phi 50$ potentiometer, Beckman, Fullerton, CA).

\section{Raw Materials and Inoculum}

Whole milk from the Linares region, Nuevo León, México, was used in all the experiments presented here. The solid fraction was increased up to $14 \%$ by enrichment with whey milk solids. The mixture was pasteurized at $60^{\circ} \mathrm{C}$ for $30 \mathrm{~min}$. No homogenization was done. Two different commercial formulations containing Streptococcus thermophilus and Lactobacillus bulgaricus were used as the inoculum: (a) a freeze-dried formulation from Distribuidora Alcatraz (culture MY800-2U, Distribuidora Alcatraz, México City, México), and (b) a freeze-dried formulation from Mconcorde-Altecsa (culture YO 814/Q, Mconcorde-Altecsa, México City, México).

\section{Inoculation Strategies}

Two different inoculation strategies were used. The first, designed to avoid the occurrence of a lag phase, consisted of dispersing $0.1 \mathrm{~g}$ of the commercial inoculum in $500 \mathrm{~mL}$ of pasteurized milk. This broth was incubated at $40^{\circ} \mathrm{C}$ in nonagitated conditions until a $\mathrm{pH}$ value of 5 was achieved. At this point, the culture, presumably still at the exponential phase, was dispersed in the entire milk volume by agitating at $200 \mathrm{rpm}$ for $5 \mathrm{~min}$. For the nonagitation fermentation experiments, agitation was interrupted 2 min after inoculum addition. The second inoculation strategy allowed for the observance of a lag phase. One gram of commercial inoculum was mixed with $10 \mathrm{~mL}$ of milk. This preparation was added to the 


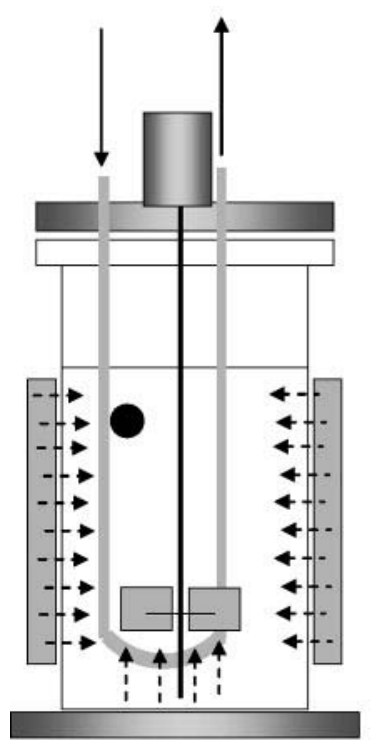

(a)

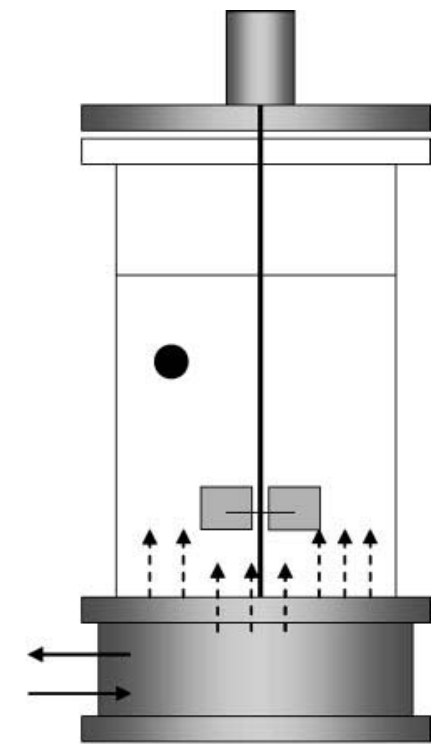

(b)
Figure 1. Experimental bioreactors. The bioreactors differed greatly in their heat-transfer characteristics: (a) 3-L fermentor, heated through the walls by an electric mantle and cooled by water circulation through a loop, and (b) 5-L fermentor, heated through a jacketed metal-dished bottom. Arrows indicate the direction of heat transfer. The black dots $(\bullet)$ indicate the location of the corresponding $\mathrm{pH}$ electrode.

fermentor. Inoculum was dispersed through agitation at $200 \mathrm{rpm}$ for $5 \mathrm{~min}$ in the entire milk volume.

\section{Fermentation Tanks}

Fermentation experiments were conducted in 2 types of bioreactors: (a) a fully instrumented 3-L ez-Control bioreactor (Applikon, Schiedam, the Netherlands), equipped with baffles, 2 Rushton turbines, a $\mathrm{pH}$ electrode, an automatic temperature control achieved by a dual system, a hot mantle covering the fermentor glass walls, and circulation of cold water through an immersed stainless steel loop (see Figure 1a), and (b) an instrumented 5-L Bioflow 3000 fermentor (New Brunswick Scientific, Edison, NJ), equipped with baffles, 2 Rushton turbines, a $\mathrm{pH}$ electrode, and an automatic temperature control achieved by hot-cold water circulation through the jacketed stainless steel reactor bottom (see Figure 1b).

\section{Acidity and $\mathrm{pH}$ Evaluations}

The $\mathrm{pH}$ was continuously monitored with the bioreactor $\mathrm{pH}$ electrode and, for the nonagitated yogurt, also from samples taken at different reactor locations by using an external electrode. For some of the experiments reported here, lactic acid concentration was measured by an on-line Fourier transform infrared (FTIR) technique, using a Mettler Toledo unit (Mettler Toledo, Columbus, $\mathrm{OH}$ ). Dissociated and nondissociated lactic acid concentration were correlated to the FTIR value of the absorbance peaks at 1,575 and $1,725 \mathrm{~cm}^{-1}$, respectively (see Hayakawa et al., 2000). The FTIR absorbance readings were calibrated by using lactic acid dilutions in the range of 1 to $15 \mathrm{~g} / \mathrm{L}$, at different $\mathrm{pH}$ values relevant to the fermentation experiments. Knowing the degree of dissociation expected at different $\mathrm{pH}$ values, calibration curves can be constructed for both the dissociated and nondissociated lactic acid forms. To calculate total lactic acid production, a regression curve was adjusted to the experimental points for dissociated and nondissociated lactic acid. From this analysis, curves of total lactic acid were constructed by addition, after proper normalization according to calibration curves.

\section{Lactose Concentration Determination}

Lactose concentration during fermentation was determined by an on-line FTIR technique, using a Mettler Toledo AutoChem unit. Lactose concentration was correlated to the FTIR value of the absorbance peaks at $1,042 \mathrm{~cm}^{-1}, 1,075 \mathrm{~cm}^{-1}$, or both (see Hayakawa et al., 2000).

\section{Population Dynamics Studies}

One-milliliter-volume samples were taken from the fermentor at different processing times to monitor the population dynamics. Streptococcus thermophilus and $L$. bulgaricus populations were estimated independently. The $S$. thermophilus population was evaluated by plating diluted samples on M17 agar ( $\mathrm{pH}$ 6.5, from Merck, Whitehouse Station, NJ) and incubating at $37^{\circ} \mathrm{C}$ for 48 h. The number of $L$. bulgaricus bacteria was estimated by plating diluted samples in de Man, Rogosa, Sharpe agar (MRS, $\mathrm{pH}$ 5.2, from Merck) and incubating at $42^{\circ} \mathrm{C}$ for $72 \mathrm{~h}$. (as described in Dave and Shah, 1996; Tharmaraj and Shah, 2003; Chammas et al., 2006). Standard counting techniques by consecutive dilutions and anaerobic incubation were used for both bacteria.

\section{Specific Growth Rate Estimations}

Specific microbial growth rates for L. bulgaricus and $S$. thermophilus were calculated from the slopes of the linear segments of plots of $\ln \left(\mathrm{x} / \mathrm{X}_{\mathrm{o}}\right)$ versus time from each batch experiment, where $\mathrm{X}$ is the population of $L$. bulgaricus or $S$. thermophilus at a given time, and $\mathrm{X}_{0}$ is the population immediately after inoculation. This 
procedure assumes a first-order growth model for each microorganism: $\mathrm{dX} / \mathrm{dt}=\mu \mathrm{X}$, where $\mathrm{t}$ is time, and $\mu$ is the specific growth rate.

\section{Viscosity and Sensory Evaluations}

The viscosity of agitated and nonagitated yogurt samples refrigerated at $4^{\circ} \mathrm{C}$ was evaluated by a Brookfield DV-II+ PRO viscometer, model RVDVII +P (Brookfield, Middleboro, MA). Samples from agitated samples were evaluated at $100 \mathrm{rpm}$ (readings fell between 38 and $40 \%$ of the torque range for that particular spindlespeed combination) by using a Brookfield RV2 spindle, and samples from nonagitated experiments were evaluated at $5 \mathrm{rpm}$ (readings fell between 50 and $51 \%$ of the torque range for that spindle-speed combination). A discriminatory triangular sensory analysis study was conducted to establish statistically whether the flavor and odor of the yogurt produced under agitated and nonagitated conditions were dissimilar (see, for example, Roessler et al., 1948). Sixty-six triangle tests were performed by 22 nontrained panelists, who were asked to establish which sample, from 3 presented, was different in flavor. The same panel analyzed raw yogurt samples (no sugar added) and yogurt samples with added honey ( $25 \mathrm{~g}$ of honey/L of yogurt) to evaluate the effects of changes in the type of sweetener on the overall sensory perception of differences among the products.

\section{RESULTS AND DISCUSION}

\section{Temperature and pH Gradients in Nonagitated Fermenting Systems}

Although apparently simple in concept, the biological process of fermenting milk into yogurt is highly complex in nature. While the temperature is set at a value between 30 and $40^{\circ} \mathrm{C}$, preferably the latter, the $\mathrm{pH}$ is allowed to descend freely as lactose is consumed and lactic acid is formed because of the action of lactic acid strains (typically no more than 2). As pH changes naturally, bacterial growth, lactose consumption, and lactic acid production rates also change with time. To complicate this already time-dependent scenario, in traditional yogurt manufacture, mixing is not applied during fermentation, as stated previously. This poses a challenge in terms of heat and mass transfer. Temperature gradients within the fermentation vessel will prevail in time, because there is no mechanism for the convective homogenization in a typically highly viscous medium. The establishment and magnitude of these gradients strongly depend on the vessel geometry. Figure 1 shows the geometrical features of the 2 bioreactors used for the experiments. The systems depicted vary greatly in their heat-transfer characteristics. The arrows within each vessel indicate the heat-transfer flux from the heating surfaces.

In the first experimental system (see Figure 1a), heat transfer is achieved by the combined action of an electrical mantle covering the tank wall and a recirculation loop through which cold water is circulated. In this system, moderate gradients are expected. Heat transfer is conducted transversely through a relatively extensive surface area. However, because the contents of the bioreactor are static, heat transfer should be far from efficient, particularly in a progressively more viscous culture medium (as a consequence of gel formation). Figure 2a presents temperature and $\mathrm{pH}$ profiles measured at 3 different radial locations within the liquid bulk ( $\mathrm{pH}$ readings from the bioreactor electrode are also presented). In a radial distance of less than $7 \mathrm{~cm}$, variations from 39 to $44^{\circ} \mathrm{C}$ were registered. Because different temperatures induce different growth and lactic acid production rates, temperature gradients induce $\mathrm{pH}$ gradients (see Figure 2a). In some cases, at the same time point, $\mathrm{pH}$ values vary by as much as 0.5 units at different radial locations in the system. Indeed, temperature differences cause different $\mathrm{pH}$ profiles at different tank locations, as clearly indicated in Figure $2 a$.

Figure 1b shows the geometrical features of the second bioreactor used in our experiments. In this case, heat is transferred from the jacketed stainless steel bottom to the liquid bulk. For this tank geometry, agitation is crucial to guarantee adequate mass and heat transfer. Because the only heat-transfer surface is located at the tank bottom, practically all the liquid surface is exposed to ambient temperature. Therefore, there is an effective heat flux through the entire height of the liquid column, approximately $20 \mathrm{~cm}$. In addition, heat is lost through the glass tank walls. Consequently, for a temperature set point of $40^{\circ} \mathrm{C}$ (temperature sensed at a single point), a strong vertical temperature gradient is expected to occur. Figure $2 \mathrm{~b}$ presents the temperature and $\mathrm{pH}$ values read at different vertical positions within the fermentor (top, medium stage, and bottom). In the first $3 \mathrm{~h}$ of processing, temperature gradients were practically nonexistent. As the fermentation began and the viscosity increased progressively because of the gel formation induced by lactic acid production, heat transfer slowed, and an increasingly wide range of temperature variation was observed. In particular, at the sampling location closer to the tank bottom, temperatures as high as 70 or $80^{\circ} \mathrm{C}$ were recorded after 4 and $5 \mathrm{~h}$ of fermentation. For instance, for the experiment presented in Figure 2b, after $5 \mathrm{~h}$ of fermentation, the temperatures at the top and bottom of the tank were 30 and $80^{\circ} \mathrm{C}$, respectively, a gradient of $50^{\circ} \mathrm{C}$ (see 
Figure $2 \mathrm{~b}$ ). This strong gradient was capable of favoring convective movement, even in this viscous environment. This phenomenon was also observed in Figure 2b. At the bottom sampling location, drastic temperature fluctuation was observed from the third to the fifth hour of processing time $\left(70,33\right.$, and $\left.80^{\circ} \mathrm{C}\right)$. The temperature dropped to $33^{\circ} \mathrm{C}$ when material from the top of the tank moved massively to the bottom, caused by the density gradient induced by the temperature differences.

Clearly, the existence of heterogeneity in this system has important process implications. For example, in yogurt fermentors, the process end-point decision is normally based on the monitoring of $\mathrm{pH}$ at a single location. Similarly, temperature control is based on measurement at a single point (rarely in more than 2 points). Interestingly, as we have illustrated here, those $\mathrm{pH}$ and temperature readings were not necessarily representative of the system.

In an agitated system, gradients are attenuated significantly. In the next sections, we compare, by using different metrics, the nonagitated and agitated fermentation processes in both of the geometries presented above. We explore and discuss the differences observed in $\mathrm{pH}$ profiles, lactic acid production, and lactose consumption profiles observed in agitated and nonagitated fermentation experiments. In addition, we discuss the differences in microbial population dynamics induced by the changes that agitation might induce in the cellular microenvironment.

\section{Process Time and Global pH Profiles}

Figure 3 presents the average $\mathrm{pH}$ gradients observed under different experimental conditions. In Figure 3a, data from 4 different experiments are presented. For all these experiments, inoculum was a powdered mixture consisting of approximately $8.88 \times 10^{6} \mathrm{cfu}$ of $S$. thermophilus and approximately $2.24 \times 10^{5}$ cfu of L. bulgaricus. The data in black correspond to nonagitated conditions (traditional process). All other conditions being the same, the data in gray correspond to agitated fermentations. In the agitated conditions, the end point was achieved approximately $1 \mathrm{~h}$ earlier. When curves were shifted in the x-axes, such that they coincided at their end point $(\mathrm{pH}=4.5)$, all slopes were practically equivalent. Therefore, no time difference was observed during the exponential phase (defined in terms of $\mathrm{pH}$ observance), and the economy of the global processing time could be attributed solely to a reduction in the lag phase. Figure 3b presents the results of 6 additional experiments under nonagitated and agitated conditions. In these experiments, inoculation was performed with $500 \mathrm{~mL}$ of yogurt in the exponential phase. Through this strategy, the lag phase was avoided. Again, when
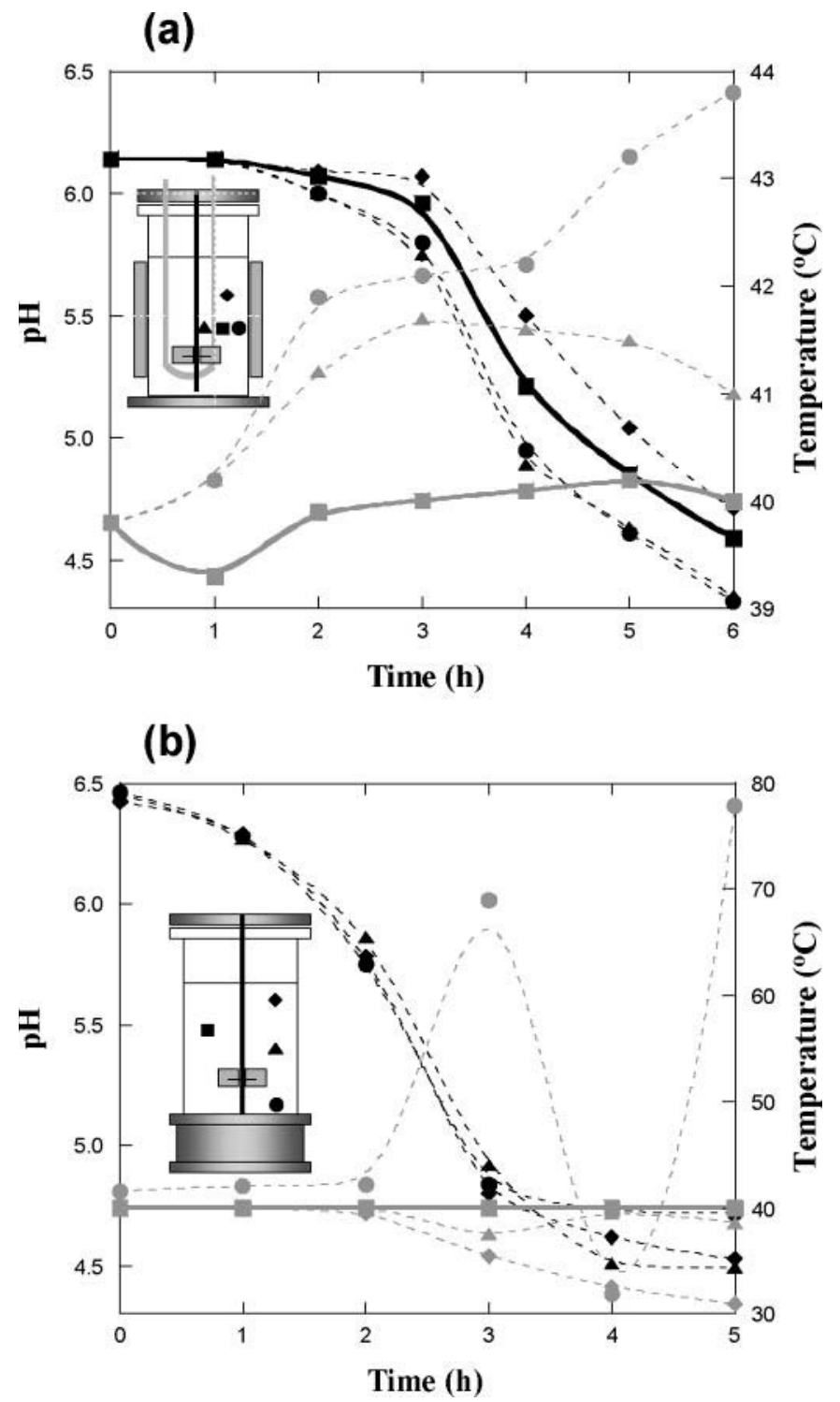

Figure 2. Temperature (gray symbols) and pH profiles (black symbols) observed within the experimental bioreactors analyzed: (a) the mantle-heated system, and (b) the bottom-jacketed system. Trends corresponding to different sampling locations are marked with specific shapes (rhombus, square, circle, triangle); sampling locations are indicated with the same symbols in the bioreactor schemes presented in the graphs. Solid lines represent trends registered by the $\mathrm{pH}$ or temperature sensors built into the bioreactor.

only the exponential phases were compared, the $\mathrm{pH}$ profile in time was practically equivalent for the agitated and nonagitated conditions. Indeed, the $\mathrm{pH}$ curve observed in our experiments for both the agitated and nonagitated conditions at $40^{\circ} \mathrm{C}$ exhibited the typical shape reported elsewhere [Hadadji et al. (2005) reported similar profiles for nonagitated conditions at $42^{\circ} \mathrm{C}$; see also De Brabandere and De Baerdemaeker, 1999; Lee and Lucey, 2004]. 


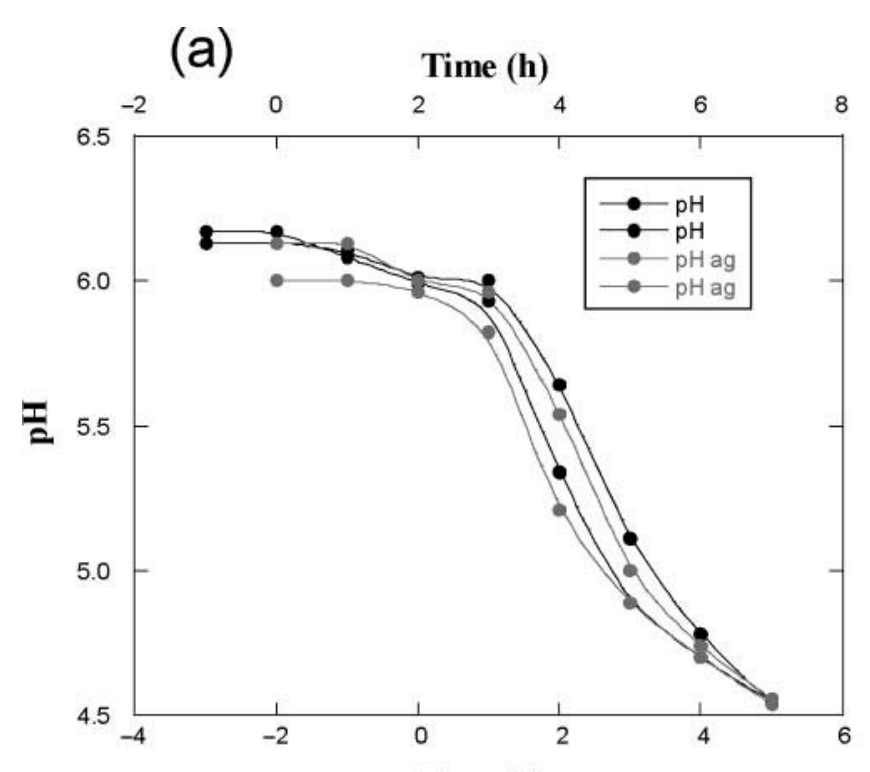

(b)

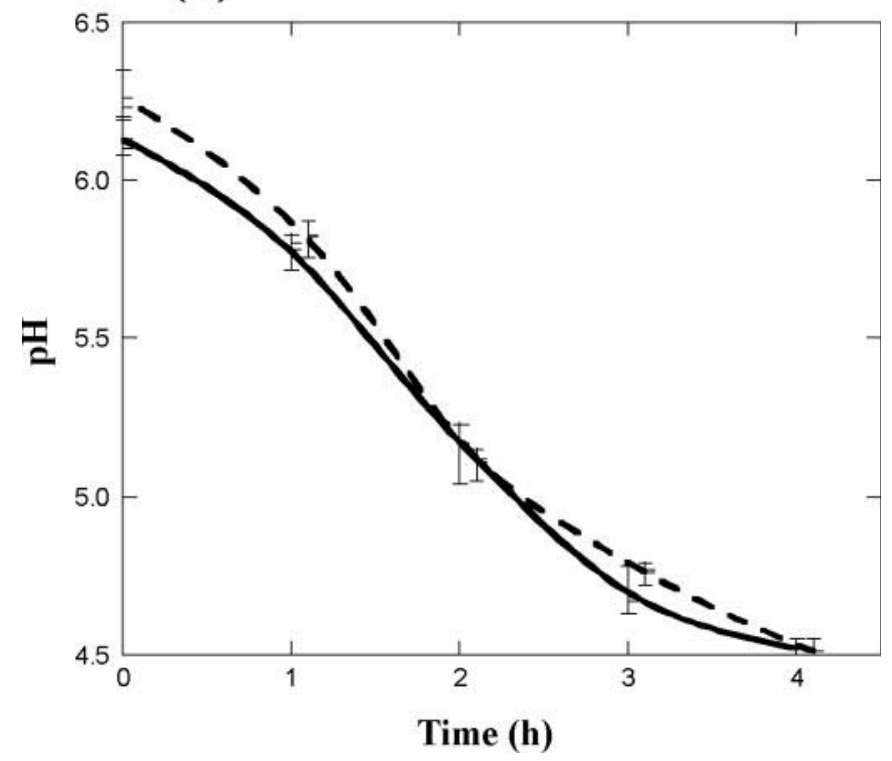

Figure 3. Evolution of $\mathrm{pH}$ with time during yogurt elaboration under different experimental conditions: (a) Fermentations that were inoculated by addition of a freeze-dried lactic acid bacterial culture and with no agitation (black circles), or with agitation at $200 \mathrm{rpm}$ (gray circles). Curves were shifted in the x-axis to overlap their exponential phases; (b) fermentations that were inoculated by addition of liquid inoculum in the exponential phase. Results from the experiments with no agitation (solid line) and with agitation at $200 \mathrm{rpm}$ (dashed line) are presented. Error bars are based on 3 repetitions.

\section{Effect of Agitation on Lactic Acid Production}

Industrial practitioners commonly believe that agitation during fermentation might inhibit lactic acid production (Driessen, 1998; Early, 2000). Agitation could enhance oxygen mass transfer from the headspace to the bulk of the liquid, creating a less anaerobic environment (microaerophilic) for fermentation, further retarding fermentation times and disrupting the ecological equilibrium. Our results confirmed these concerns only partially and showed that the agitation values tested here did not significantly alter the observed $\mathrm{pH}$ or global lactic acid profiles during fermentation. However, significant differences were observed in the concentration profiles of dissociated and nondissociated lactic acid in experiments at the 2 different agitation conditions tested. Figure 4 shows the profiles in time for the production of lactic acid in the agitated and nonagitated fermentations, obtained by online FTIR measurement (experiments performed in the fermentor shown in Figure 1b). Immediately after inoculation, lactic acid production began and followed a rapid reaction rate (see Figure 4a). For the agitated conditions, the initial rate of production of lactic acid (initial slope of the curves in Figure 4a) was greater. This result is consistent with the shorter lag phase observed in $\mathrm{pH}$ profiles of the agitated fermentations discussed above. Indeed, the global lactic acid profiles for the nonagitated yogurt were delayed by approximately $1 \mathrm{~h}$, an observation consistent with the similar delay in $\mathrm{pH}$ profiles. Later during the fermentation, the lactic acid production rate slowed down until a final lactic acid concentration was achieved.

The FTIR technique allowed for the monitoring of both the dissociated and nondissociated forms of lactic acid (following the absorbance peak signals at 1,575 and $1725 \mathrm{~cm}^{-1}$, respectively). During yogurt fermentation, as the $\mathrm{pH}$ value changed, the lactic acid equilibrium shifted progressively. Monitoring both lactic acid forms independently might provide insight into this shift. Figure $4 \mathrm{~b}$ presents the online experimental determinations of dissociated and nondissociated lactic acid concentrations for the agitated and nonagitated fermentations. The concentration profiles for both acid forms were significantly different. Remarkably, the nondissociated lactic acid production rate was clearly faster in the agitated fermentations. At all times, the nondissociated lactic acid concentration was greater in the experiments using agitation. The concentration ratios between the nondissociated and dissociated acid forms were modified because of agitation (see Figure 4c), reaching a maximum value of 2.7 for the agitated systems and a maximum of only 1.8 for the nonagitated experiments. It is well known that the nondissociated acid lactic form is responsible of the inhibitory effect of lactic acid bacteria against themselves and other bacteria (Piard and Desmazeaud, 1991). Therefore, a greater growth inhibitory effect was expected to take place in agitated fermentations. This inference is consistent with an observation discussed below in greater detail: a greater degree of growth inhibition was observed in the agitated conditions, particularly for L. bulgaricus. 


\section{Effect of Agitation on Population Dynamics Depends on Geometry}

Significant differences were observed in the ecological dynamics occurring in agitated versus nonagitated yogurt fermentation. According to our results, the extent and nature of these differences did not appear to be universal, but rather appeared to be a strong function of the fermentation vessel geometry. More specifically, as expected, greater differences between the agitated and nonagitated fermentations were observed in the fermenting system in which the geometry induced greater temperature gradients because of heat-transfer limitations.

Figure 5 presents the results from the nonagitated (Figure 5a) and agitated (Figure 5b) fermentation experiments carried out in the vessel described in Figure 1a. As discussed previously, temperature gradients within this system existed, but were not as drastic as those observed in the fermentor system portrayed in Figure 1b (results analyzed later in this section). Average $\mathrm{pH}$ profiles, and $S$. thermophilus and L. bulgaricus concentration profiles are presented. In general, the behavior described concurs with the classical notion of ecological succession for milk fermentative systems: $S$. thermophilus acidifies the environment, making it progressively more suitable for L. bulgaricus growth. It is well documented and accepted (see, for example, Shah, 2000; Mahdian and Mazaheri-Tehrani, 2007) that during yogurt making, $S$. thermophilus dominates the early stage of yogurt fermentation. As the redox potential of milk medium is reduced and the $\mathrm{pH}$ is lowered from 6.5 to 5.5 , the growth of $L$. bulgaricus is stimulated. Although throughout the fermentation process the $S$. thermophilus population is always greater than the $L$. bulgaricus population, below $\mathrm{pH} 5.0$ the L. bulgaricus specific growth rate dominates yogurt fermentation and produces acetaldehyde and additional lactic acid, yielding the "green apple" flavor characteristic of yogurt and further decreasing $\mathrm{pH}$ values to near 4.6, progressively inducing clotting and gelatinization. However, the synergistic relationship of $L$. bulgaricus with $S$. thermophilus in mixed yogurt cultures is more complex and has been well documented (Moon and Reinbhold, 1976; Rajagopal and Sandine, 1990; Riva et al., 1997). For example, the amino acids arising from the proteolytic activity of $L$. bulgaricus have been identified as specific growth stimulants for Streptococcus spp. strains (Galesloot et al., 1968; Veringa et al., 1968). In general, nonagitated and agitated fermentations follow similar trends, but some particularities should be mentioned. For the nonagitated fermentations (Figure $5 a)$, an apparently longer lag phase was observed. In the agitated fermentations, the exponential change of (a)

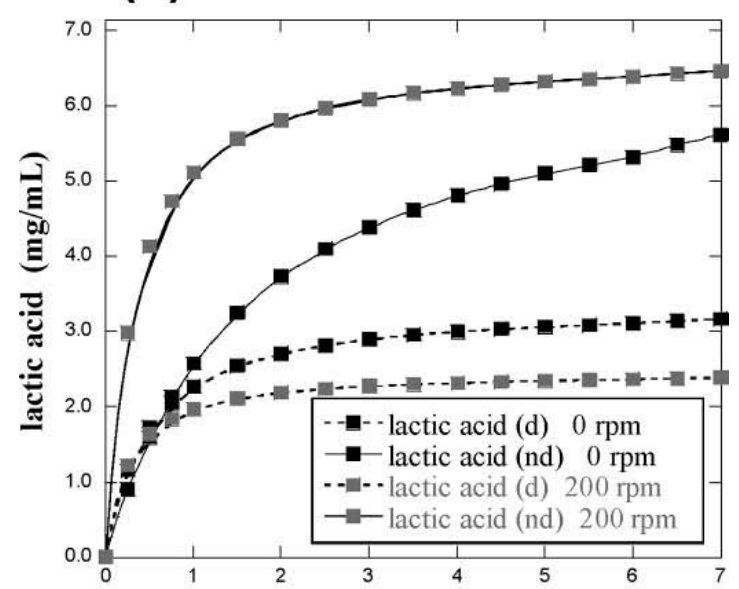

(b)

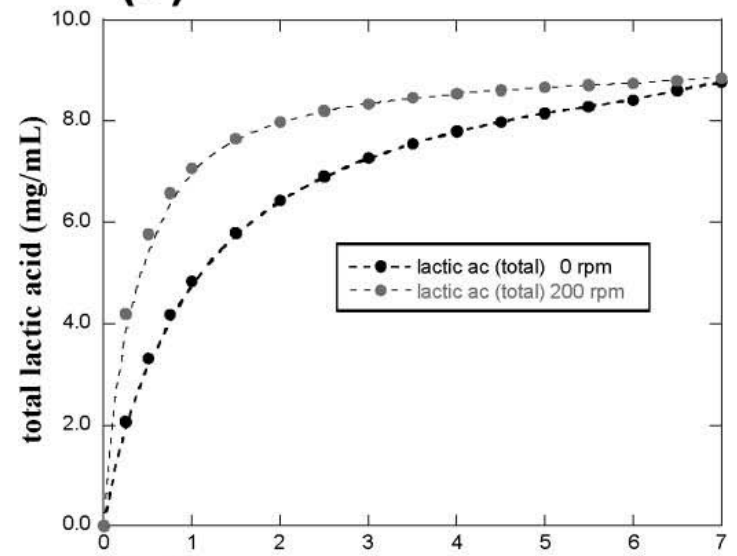

(c)

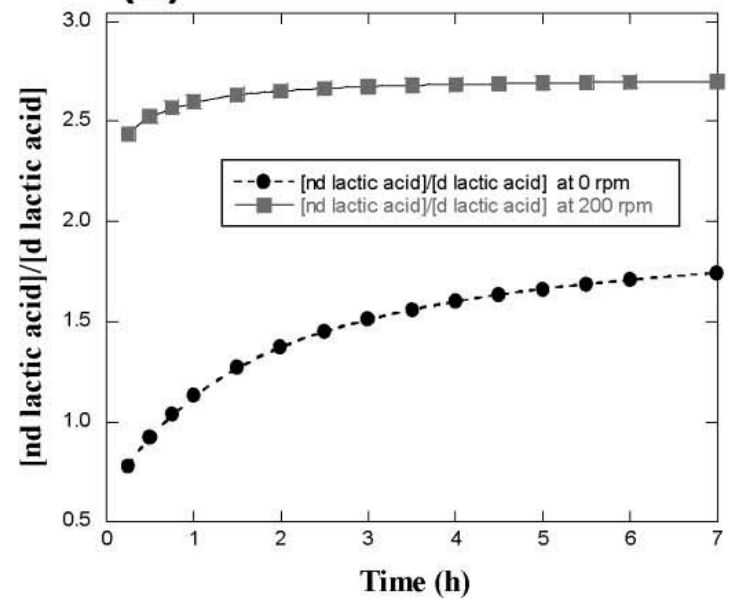

Figure 4. Lactic acid profiles (expressed as mg of lactic/mL) observed during elaboration of liquid yogurt under agitated and nonagitated fermentation experiments: (a) dissociated (dashed line) and nondissociated (solid line) lactic acid profiles in nonagitated (black squares) and agitated (gray squares) fermentation experiments, (b) global lactic acid profiles in nonagitated (black circles), and agitated (gray circles) fermentation experiments, (c) nondissociated (nd)/dissociated (d) lactic acid concentration ratios in nonagitated (black circles) and agitated (gray squares) fermentation experiments. 
(a)

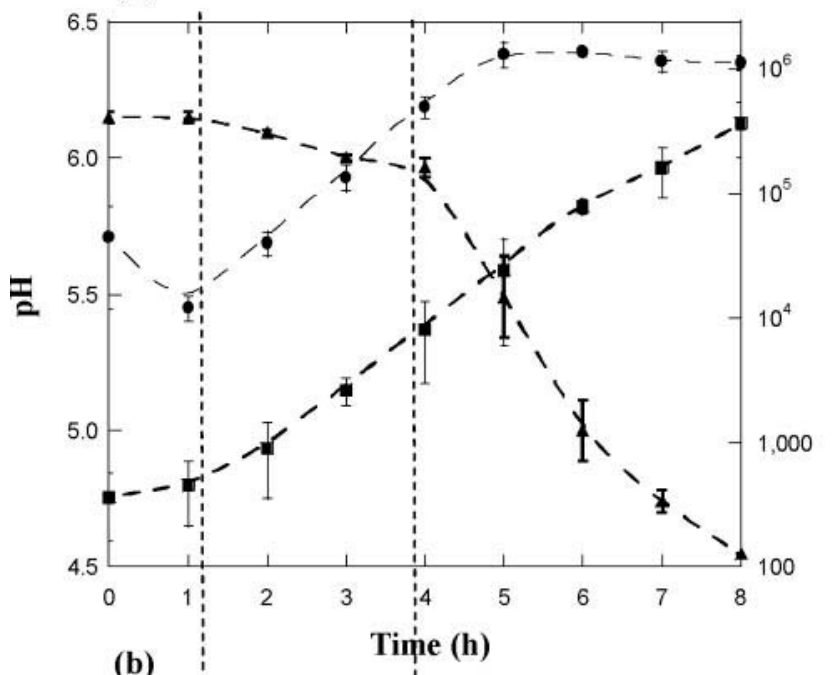

(b)

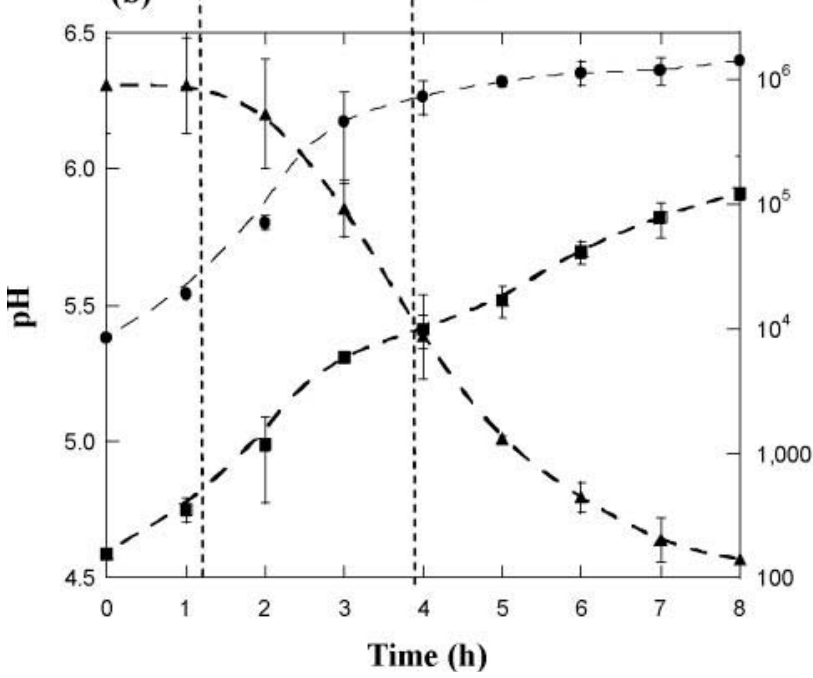

Figure 5. The pH profiles ( $\mathbf{\Delta})$ and population dynamics of Streptococcus thermophilus $(\mathbf{)})$ and Lactobacillus bulgaricus (ם; expressed as $\times 10^{3} \mathrm{cfu} / \mathrm{mL}$ ) during the elaboration of yogurt under (a) nonagitated and (b) agitated conditions. Error bars are based on 3 repetitions.

$\mathrm{pH}$ and of $S$. thermophilus and L. bulgaricus counts occurred earlier. However, in both the traditional and agitated fermentations, the slopes of decrease in $\mathrm{pH}$ and microbial growth were very similar during the first hours of the exponential phase. After approximately $4 \mathrm{~h}$, the growth curve for $S$. thermophilus reached its plateau in the agitated fermentations, $1 \mathrm{~h}$ faster than in the nonagitated fermentations. This is also consistent with the earliest occurrence of the exponential phase in the agitated experiments. Despite this displacement in time, the population growth curve for S. thermophilus reached the same maximum value, for a final population of slightly greater than $10^{9}$ cells $/ \mathrm{mL}$.

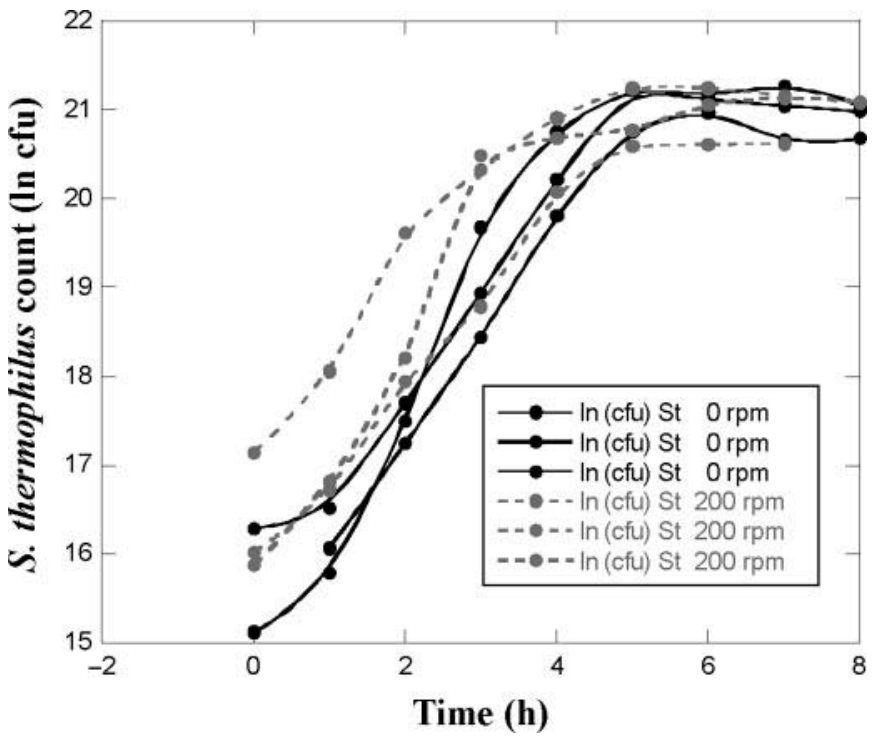

Figure 6. Growth curves of Streptococcus thermophilus [expressed as $\ln (\mathrm{cfu} / \mathrm{mL})$ ] during the elaboration of liquid yogurt in experiments at $40^{\circ} \mathrm{C}$ with no agitation (black circles, solid line), and with agitation at $200 \mathrm{rpm}$ (gray circles, dashed line). Experiments conducted in the bioreactors shown in Figure 1a and Figure 1b are plotted. If curves were shifted in the $\mathrm{x}$-axis, they would collapse into a master curve, implying practically equivalent kinetic behaviors regardless of agitation conditions.

Figure 6 presents $S$. thermophilus growth curves in nonagitated and agitated fermentations. Experiments in both the mantle-heated and bottom-heated fermentors were included. It can be concluded, based on these results, that in the range of experimental conditions explored here, the $S$. thermophilus growth behavior was robust at $40^{\circ} \mathrm{C}$, regardless of the agitation and system geometry characteristics. The population curves were practically equivalent under the agitated and nonagitated conditions. In Figure 6, the population is plotted in a natural logarithmic scale [as $\ln$ (population in cfu)]. Therefore, the slopes from the different curves directly reflect the specific growth rate of $S$. thermophilus in exponential growth. Remarkably, the slopes from the agitated and nonagitated fermentations were also practically equivalent.

For L. bulgaricus, the population behavior differed in the nonagitated and agitated fermentations. After approximately $4 \mathrm{~h}$ of fermentation, a slight deceleration was noticeable for the agitated fermentations (see the inflection in the L. bulgaricus growth curve in Figure 5b compared with Figure 5a). Indeed, the final L. bulgaricus population values for the nonagitated fermentations were, reproducibly, 3 times greater than for the agitated fermentations (at the same $\mathrm{pH}$ value). This observation is consistent with the greater nondissociated lactic acid concentrations prevalent in the agitated fermentations 


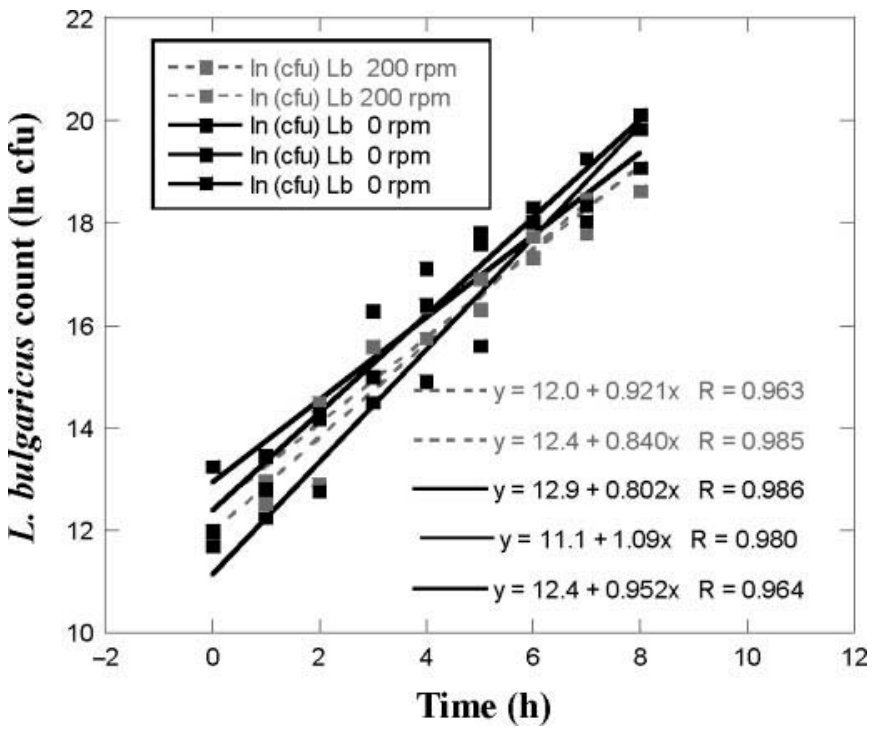

Figure 7. Growth curves of Lactobacillus bulgaricus [expressed as $\ln (\mathrm{cfu} / \mathrm{mL})]$ during the elaboration of liquid yogurt in experiments at $40^{\circ} \mathrm{C}$ with no agitation (black squares, solid line), and with agitation at $200 \mathrm{rpm}$ (gray squares, dashed line). Only experiments performed in the mantle-heated bioreactor (see Figure 1a) are plotted. Experimental data points were adjusted to linear trends, and the resulting fitted equations are presented.

(Figure 4), arguably inducing growth inhibition in the late growth phase of $L$. bulgaricus. Figure 7 summarizes the growth curves for L. bulgaricus from 6 agitated and nonagitated fermentation experiments (only for cases run in the mantle-heated systems). Because the population was plotted as $\ln$ (population in cfu) versus time, the slope of each curve is the specific growth rate of $L$. bulgaricus in the exponential phase (slopes presented in Figure 7). On average, slope values for the nonagitated fermentations were slightly (but statistically not significantly) greater. Therefore, a clear effect on the exponential specific growth rate was not conclusive. Again, in all cases, final population values were always greater for nonagitated cases. Figure 8 provides more insight into the nature of the previously stated inhibition effect over $L$. bulgaricus. Two experiments were contrasted, a nonagitated and an agitated fermentation, otherwise conducted under equivalent conditions. Lactose consumption (as measured by online FTIR) and L. bulgaricus population [plotted as $\ln$ (population in $\mathrm{cfu}$ )] are plotted in the same graph. Lactobacillus bulgaricus breaks each mole of lactose into 1 mol of galactose and $1 \mathrm{~mol}$ of glucose, to later take glucose as its energy source for microbial growth. Therefore, and as observed in Figure 8, lactose consumption and biomass growth are intrinsically coupled. However, several clear differences between the agitated and the nonagitated fermentations should be emphasized. The lactose con-

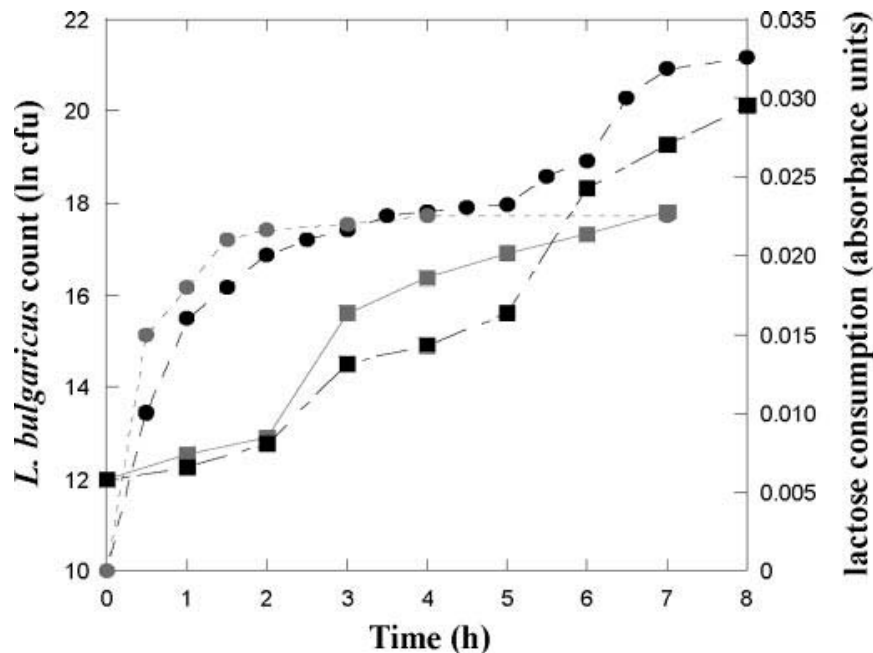

Figure 8. Relationship between lactose consumption (circles) and Lactobacillus bulgaricus growth [squares; expressed as $\ln (\mathrm{cfu} / \mathrm{mL})$ ] during the elaboration of liquid yogurt in experiments at $40^{\circ} \mathrm{C}$ with no agitation (black circles and squares) and with agitation at $200 \mathrm{rpm}$ (gray circles and squares).

sumption curves were remarkably similar, and a first plateau was achieved after $3 \mathrm{~h}$. Although no further growth or lactose consumption was observed for the remaining fermentation time for the agitated fermentations, a second curve of lactose consumption, associated with consequent $L$. bulgaricus growth, occurred for the nonagitated fermentations. Although in the agitated fermentations, high nondissociated lactic acid concentrations inhibited the occurrence of a second lactose consumption period, in the nonagitated fermentations, significantly lower nondissociated lactic acid concentrations (see Figure $4 \mathrm{~b}$ and $4 \mathrm{c}$ ) still allowed for further lactose degradation and L. bulgaricus growth.

Figure 9 presents estimations of the microbial population profiles of $S$. thermophilus and L. bulgaricus in experiments performed in the fermentor depicted in Figure 1b. Regardless of the agitation conditions, the concentration profiles of $S$. thermophilus were consistent among all the experiments. Note that from the series of 6 experiments presented, the final population of $S$. thermophilus remained controlled in a narrow band around $10^{9}$. Indeed, the final population counts for $S$. thermophilus in these experiments, and the general shape of the population curve, were also similar to those observed for the same microorganism in agitated and nonagitated fermentation experiments conducted in the mantle-heated vessel geometry (Figure 1a). However, $L$. bulgaricus population curves were significantly different in the agitated and nonagitated fermentations. With $40^{\circ} \mathrm{C}$ being the optimum temperature for $L$. bulgaricus growth, deviations from this condition attributable to bioreactor heterogeneities imply deviations with respect 


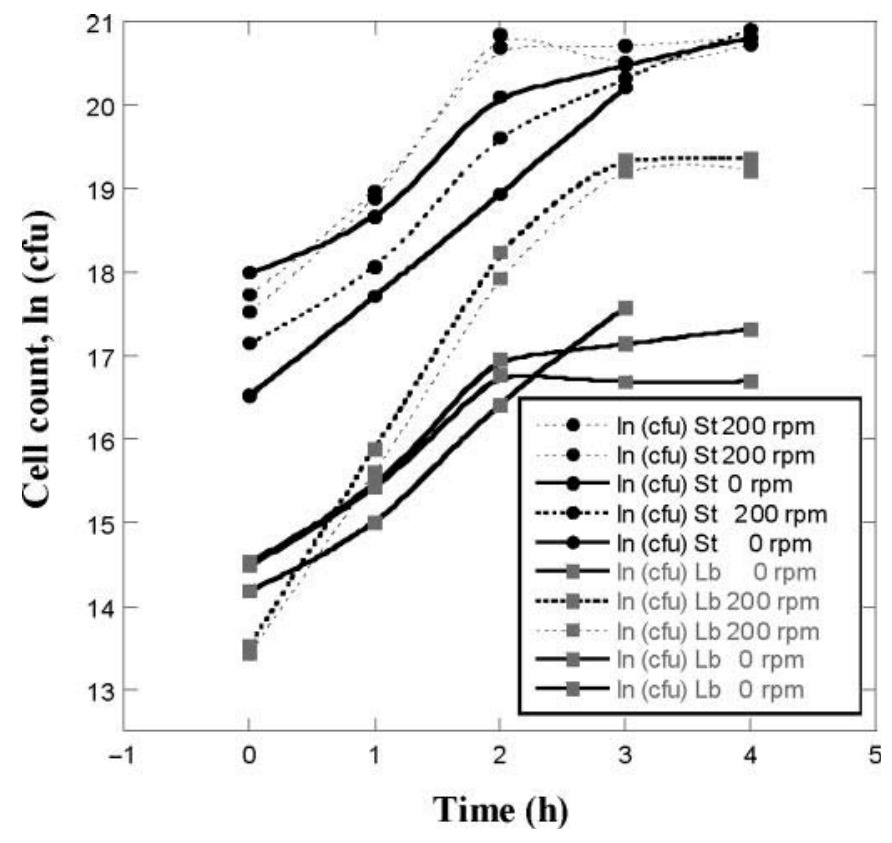

Figure 9. Growth curves of Streptococcus thermophilus (circles) and Lactobacillus bulgaricus (squares; expressed as $\times 10^{3} \mathrm{cfu} / \mathrm{mL}$ ) during the elaboration of liquid yogurt in experiments at $40^{\circ} \mathrm{C}$ with no agitation (solid lines) and with agitation at $200 \mathrm{rpm}$ (dashed lines). Only experiments performed in the bottom-jacketed bioreactor (see Figure 1b) are plotted.

to ideal growth. Within this fermentor system, as shown in Figure 2b, important temperature gradients existed in nonagitated conditions, particularly in the vertical direction. At some times, specifically at the end of the fermentation, the vertical temperature gradients were enhanced by the increasing heat-transfer resistance caused by the progressively more viscous medium. Temperatures in locations closer to the liquid surface could have temperatures in the range of 30 to $35^{\circ} \mathrm{C}$, far from the optimum growth condition for L. bulgaricus. In addition, the culture media at the tank bottom experienced high temperatures, inclusively above pasteurization values. Table 1 presents specific growth rates $[\mu$ $=\mu(\mathrm{T}, \mathrm{pH})]$ for both $S$. thermophilus and L. bulgaricus calculated from the exponential growth phase of agitated and nonagitated fermentations according to the protocol already described. In all cases, $\mu$ was calculated rigorously and exclusively from the linear region of plots of $\ln \left(\mathrm{X} / \mathrm{X}_{\mathrm{o}}\right)$ versus $\mathrm{t}$. The average values for the specific growth rate of $S$. thermophilus were 0.997 to $1.056 / \mathrm{h}$ from the nonagitated and agitated conditions, respectively. No statistically significant differences existed between these values. However, the average specific growth rate for L. bulgaricus more than doubled because of agitation, from 1.032 to $2.30 / \mathrm{h}$, when the nonagitated and agitated fermentations at $40^{\circ} \mathrm{C}$ were compared.

\section{Effects of Agitation on the Viscosity and Sensory Characteristics of the Final Product}

As expected, gel formation was affected by agitation. Viscosity values for the agitated and nonagitated samples were significantly different $(P<0.05)$. The average viscosity values from 3 independent agitated experiments were 158.0, 150.0, and $152 \mathrm{cP}$. In contrast, samples from the nonagitated fermentation experiments presented viscosity values of 4,088, 4,052, and $4,096 \mathrm{cP}$. In addition, the degree of syneresis, evaluated as whey losses $(\mathrm{g})$ during storage, was greater in the agitated yogurt treatments. On average, nonagitated samples released $15 \mathrm{~g}$ of whey/100 g of product after 3 $\mathrm{d}$ of refrigeration at $4^{\circ} \mathrm{C}$. Agitated samples released 50 $\mathrm{g}$ of whey/100 g of product under the same refrigeration conditions. Gel water retention properties might be improved by addition of a gelation agent, a common practice in commercial yogurt manufacturing. Regarding overall flavor perception, nonstatistical differences at the 0.05 level of significance were observed between samples from the agitated and nonagitated fermentations. A nonexpert sensory panel composed of 22 members performed 66 yogurt triangle tests (ASTM, 2004 ) that included samples from the agitated and nonagitated fermentations. The results indicated that at a significance level of 0.05 , the panel was unable

Table 1. Specific growth rates $\left(\mu, \mathrm{h}^{-1}\right)$ of Streptococcus thermophilus (St) and Lactobacillus bulgaricus (Lb) in nonagitated and agitated fermentation experiments conducted in the bottom-jacketed bioreactor system ${ }^{1}$

\begin{tabular}{llllllll}
\hline Condition & Moo & $\mu_{\exp 1}{ }^{3}$ & $\mu_{\exp 2}$ & $\mu_{\exp 3}$ & $\mu_{\exp 4}$ & Mean & SD \\
\hline \multirow{2}{*}{$200 \mathrm{rpm}$} & $\mathrm{St}$ & 1.067 & 1.09 & 1.107 & 0.96 & 1.056 & 0.057 \\
& $\mathrm{Lb}$ & 2.241 & 2.359 & - & - & 2.300 & 0.059 \\
0 & $\mathrm{rpm}$ & 0.889 & 0.912 & - & 1.19 & 0.997 & 0.137 \\
& $\mathrm{Lb}$ & 1.136 & 0.931 & 0.9678 & 1.095 & 1.032 & 0.085 \\
\hline
\end{tabular}

${ }^{1}$ Illustrated in Figure 1b. In this bioreactor system, severe temperature gradients were observed under nonagitated conditions.

${ }^{2} \mathrm{Moo}=$ microorganism.

${ }^{3}$ At least 2 experimental specific growth rates $\left(\mu_{\exp n}\right)$ are presented for each condition. 
to discriminate between samples correctly, despite the observed viscosity differences in the products. In a second sensory study also using triangle tests, yogurt samples were liquefied with $2 \%$ honey (wt/wt), and the panel did not find a statistical difference in the texture or flavor between samples coming from agitated or nonagitated fermentations.

\section{CONCLUSIONS}

In this contribution, we explored the effect of agitation during fermentation in yogurt manufacturing. We documented that, for the traditional nonagitated yogurt elaboration process, the population dynamics, $\mathrm{pH}$ profiles, lactic acid production, and lactose consumption profiles were strongly dependent on the geometry of the fermentation system. Specifically, this dependence was related mainly to temperature gradients that occurred and prevailed in the nonagitated fermentation systems. These gradients generated differences in local lactic acid production rates, $\mathrm{pH}$ microenvironments, local lactose consumption rates, and consequently, local differences in growth rates. In cases in which temperature gradients are severe, the heterogeneity originated might affect the global fermentation performance. Agitation during fermentation diminished system heterogeneity by significantly improving heat transfer. In our experiments, in the fermentation system with the greater potential for severe temperature heterogeneity, the global observable specific growth rate of $L$. bulgaricus was more than 2-fold greater in the agitated than in the nonagitated fermentations. In addition, the final $L$. bulgaricus population count was consistently greater in the agitated fermentations.

In our experiments in bioreactors with smaller temperature gradients occurring in nonagitated conditions, we observed that agitation during fermentation did not directly affect (either positively or negatively) intrinsic growth rates or exponential global lactic acid production rates. In fermentation experiments at low to moderate inoculum concentrations conducted under agitated conditions, an overall economy in processing time was observed because of a reduction in the lag phase (as define by $\mathrm{pH}$ changes). However, no reduction in the time of the exponential phase could be attributed to agitation.

Here, we also report an unanticipated effect of agitation. Greater nondissociated lactic acid concentrations were observed in agitated versus nonagitated fermentations. This effect is relevant and is probably related to the creation of a slightly microaerobic environment through the incorporation of small amounts of air via agitation. Nondissociated lactic acid had a greater growth inhibitory effect than its dissociated form. In- deed, some degree of growth inhibition was observed, specifically over L. bulgaricus, in our agitated fermentation experiments. This inhibition was not noticeable in fermentors with severe heat-transfer limitations, because temperature heterogeneity hindered its effect.

In terms of sensorial quality, as expected, yogurt samples agitated during fermentation had lower viscosities and poorer gel properties, which might be improved by adding a gelation agent. However, the nontrained panel distinguished no statistical difference in flavor between the agitated and nonagitated samples.

Our results suggest that agitation during fermentation to produce liquid yogurt might have practical benefits, particularly in fermentation vessels with poor heattransfer characteristics. Nowadays, traditional yogurt manufacturing is restricted to small-scale fermentation vessels, precisely because of the heat-transfer restrictions. Agitation might make it possible to perform fermentations to produce drinkable yogurt at a controlled temperature on a larger scale without sacrificing product quality attributes.

\section{REFERENCES}

Al-Homoud, A., M. Hondzo, and T. LaParra. 2007. Fluid dynamics impact on bacterial physiology: Biochemical oxygen demand. J. Environ. Eng. 133:226-236.

Albasi, C., P. Tataridis, E. S. Manjarrez, and P. Tailandier. 2001. A new tool for the quantification of microorganism interaction dynamics. Ind. Eng. Chem. Res. 40:5222-5227.

ASTM. 2004. E1885-04: Standard Test Method for Sensory AnalysisTriangle Test. ASTM International, West Conshohocken, PA.

Chammas, G. I., R. Saliba, G. Corrieu, and C. Béal. 2006. Characterisation of lactic acid bacteria isolated from fermented milk "laban." Int. J. Food Microbiol. 110:52-61.

Dave, R. I., and N. P. Shah. 1996. Evaluation of media for selective enumeration of Streptococcus thermophilus, Lactobacillus delbrueckii ssp. bulgaricus, Lactobacillus acidophilus, and Bifidobacteria. J. Dairy Sci. 79:1529-1536.

De Brabandere, A. G., and J. G. De Baerdemaeker. 1999. Effects of process conditions on the $\mathrm{pH}$ development during yogurt fermentation. J. Food Eng. 41:221-227.

Driessen, F. M. 1998. New Developments in the Manufacture of Fermented Milk Productos. Bull. No. 227. Int. Dairy Fed., Brussels, Belgium.

Early, R. 2000. Tecnología de los Productos Lácteos. Editorial Acribia, Zaragoza, Spain.

Ekinci, F. Y., and M. Gurel. 2008. Effect of using propionic acid bacteria as an adjunct culture in yogurt production. J. Dairy Sci. 91:892-899.

Elli, M., M. L. Callegari, S. Ferrari, E. Bessi, D. Cattivelli, S. Soldi, L. Morelli, N. Goupil Feuillerat, and J.-M. Antoine. 2006. Survival of yogurt bacteria in the human gut. Appl. Environ. Microbiol. 72:5113-5117.

Galesloot, Th. E., F. Hassing, and H. A. Veringa. 1968. Symbiosis in yogurt (1). Stimulation of Lactobacillus bulgaricus by a factor produced by Streptococcus thermophilus. Neth. Milk Dairy J. 22:50-63.

Hadadji, M., R. Benama, N. Saidi, D. Henni, and M. Kihal. 2005. Identification of cultivable Bifidobacterium species isolated from breast-fed infants feces in West-Algeria. Afr. J. Biotechnol. 4:422-430.

Hayakawa, K., K. Harada, S. Takeuchi, S. Shibata, and A. Miyagi. 2000. Method for controlling culture of lactic bacteria. US Patent 6054262. Issued April 25, 2000. 
Kristo, E., C. G. Biliaderis, and N. Tzanetakis. 2003. Modelling of the acidification process and rheological properties of milk fermented with a yogurt starter culture using response surface methodology. Food Chem. 83:437-446.

Kulkarni, C., N. Belsare, and A. Lele. 2006. Studies on shrikhand rheology. J. Food Eng. 74:169-177.

Lee, W. J., and J. A. Lucey. 2004. Structure and physical properties of yogurt gels: Effect of inoculation rate and incubation temperature . J. Dairy Sci. 87:3153-3164.

Mahdian, E., and M. Mazaheri Tehrani. 2007. Evaluation the effect of milk total solids on the relationship between growth and activity of starter cultures and quality of concentrated yoghurt. Am.-Euras. J. Agric. Environ. Sci. 2:587-592.

Maingonnat, J. F., L. Muller, and J. C. Leuliet. 2005. Modelling the build-up of a thixotropic fluid under viscosimetric and mixing conditions. J. Food Eng. 71:265-272.

Manjarrez, E. S., C. Albasi, and J. P. Riba. 2000. A two-reservoir, hollow-fiber bioreactor for the study of mixed-population dynamics: Design aspects and validation of the approach. Biotechnol. Bioeng. 69:401-408.

Moon, N. J., and G. W. Reinbhold. 1976. Commensalism and competition in mixed cultures of Lactobacillus bulgaricus and Streptococcus thermophilus. 1. Milk. J. Milk Food Technol. 39:337-341.

Padmasiri, S. I., J. Z. Zhang, M. Fitch, B. Norddahl, E. Morgenroth, and L. Raskin. 2007. Methanogenic population dynamics and performance of an anaerobic membrane bioreactor (AnMBR) treating swine manure under high shear conditions. Water Res. 41:134-144.

Parayre, S., H. Falentin, M. N. Madec, K. Sivieri, A. S. Le Dizes, D. Soller, and S. Lortal. 2007. Easy DNA extraction method and optimisation of PCR-temporal temperature gel electrophoresis to identify the predominant high and low GC-content bacteria from dairy products. J. Microbiol. Methods 69:431-441.

Patocka, G., R. Cervenkova, and P. Jelen. 2004. Textural effects of soluble whey protein isolate in stirred yogurt. Milchwissenschaft $59: 37-40$.

Piard, J. C., and M. Desmazeaud. 1991. Inhibiting factors produced by lactic acid bacteria. 1. Oxygen metabolites and catabolism endproducts. Lait 71:525-541.

Rajagopal, S. N., and W. E. Sandine. 1990. Associative growth and proteolysis of Streptococcus thermophilus and Lactobacillus bulgaricus in skim milk. J. Dairy Sci. 73:894-899.

Riva, M., L. Franzetti, A. Galli, and A. Schiraldi. 1997. Growth and fermentation activity of Streptococcus thermophilus and Lactobacillus delbrueckii subsp. bulgaricus in milk: A calorimetric investigation. Ann. Microbiol. Enzymol. 47:199-211.

Roessler, E. B., J. Warren, and J. F. Gwimon. 1948. Significance in triangular test. Food Res. 13:503-510.

Shah, N. P. 2000. Probiotic bacteria: Selective enumeration and survival in dairy foods. J. Dairy Sci. 83:894-907.

Tharmaraj, N., and N. P. Shah. 2003. Selective enumeration of Lactobacillus delbrueckii ssp. bulgaricus, Streptococcus thermophilus, Lactobacillus acidophilus, Bifidobacteria, Lactobacillus casei, Lactobacillus rhamnosus, and Propionibacteria . J. Dairy Sci. $86: 2288-2296$.

Veringa, H. A., T. E. Galesloot, and H. Davelaar. 1968. Symbiosis in yoghurt (2). Isolation and identification of a growth factor for Lactobacillus bulgaricus produced by Streptococcus thermophilus. Neth. Milk Dairy J. 22:114-120. 